\title{
Clinical observation of pulmonary lymphoepithelioma-like carcinoma
}

\author{
Zhou Sha ${ }^{1,2 \#}$, Yajun Wei, ${ }^{2,3}$, Tinghua Gao ${ }^{4 \#}$, Yuanling Luo ${ }^{5}$, Jiaqun Chen ${ }^{3}$, Ting Li $^{1,2}$, Liyang $\mathrm{Hu}^{1,2}$, \\ Xiaoli Niu ${ }^{6}$, Zhong Lin ${ }^{1,2}$, Weize $\mathrm{Lv}^{2,3}$, Xiaofeng Pei ${ }^{1,2}$ \\ ${ }^{1}$ Department of Thoracic Oncology, The Fifth Affiliated Hospital of Sun Yat-sen University, Zhuhai, China; ${ }^{2}$ Guangdong Provincial Key Laboratory \\ of Biomedical Imaging, The Fifth Affiliated Hospital, Sun Yat-sen University, Zhuhai, China; ${ }^{3}$ Department of Interventional Medicine, The Fifth \\ Affiliated Hospital Sun Yat-sen University, Zhuhai, China; ${ }^{4}$ Department of Oncology, First People's Hospital of Zhaoqing City, Zhaoqing, China; \\ ${ }^{5}$ Department of Head and Neck Oncology, The Fifth Affiliated Hospital of Sun Yat-sen University, Zhuhai, China; ${ }^{6}$ Department of Infectious \\ Diseases, The Fifth Affiliated Hospital of Sun Yat-sen University, Zhuhai, China \\ Contributions: (I) Conception and design: Z Sha, W Lv, Z Lin; (II) Administrative support: X Pei; (III) Provision of study materials or patients: T \\ Gao, Y Luo, J Chen, T Li, L Hu; (IV) Collection and assembly of data: Y Wei, X Niu; (V) Data analysis and interpretation: Z Sha; (VI) Manuscript \\ writing: All authors; (VII) Final approval of manuscript: All authors. \\ \#These authors contributed equally to this work. \\ Correspondence to: Weize Lv. Department of Interventional Medicine, The Fifth Affiliated Hospital Sun Yat-sen University, 52 Mei Hua East Road, \\ Zhuhai 519000, China. Email: lvweize@163.com; Xiaofeng Pei. Department of Thoracic Oncology, The Fifth Affiliated Hospital of Sun Yat-sen \\ University, 52 Mei Hua East Road, Zhuhai 519000, China. Email: pei63052161@139.com.
}

Background: Lymphoepithelioma-like carcinoma (LELC) of the lung is a rare type of non-small cell lung cancer (NSCLC), and researches of it are still not enough.

Methods: In this study, we retrospectively analyzed 36 patients with LELC diagnosed in the Fifth Affiliated Hospital of Sun Yat-sen University and Zhaoqing First People's Hospital from January 2014 to June 2021, to investigate the clinical manifestations, tumor markers, treatment, and prognosis of LELC. Clinical data including age, gender, smoking history, family history of cancers, Epstein-Barr virus (EBV) encoding RNA (EBER) status, gene mutations, programmed death-ligand 1 (PD-L1) expression, treatment, and prognosis.

Results: There was a total of 36 participants in this study, 16 males and 20 females, the median age was 57 years (37-76 years). A total of 22 cases (61.1\%) were advanced (stage III and IV), and EBER was 94.4\% positive. Most patients were treated with surgery, platinum chemotherapy, or radiotherapy. At the time of 31 June 2021, 33 participants had survived, and the longest survival time was 72 months. Lung LELC was more common in old participants ( $\geq 59$ years) and was not associated with smoking history. Expression of PD-L1 was positive in the majority ( 27 cases, $75 \%$ ) and participants with positive PD-L1 expression tended to have longer progression-free survival (PFS) and overall survival (OS) time than those with negative PD-L1 expression.

Conclusions: Pulmonary LELC usually occurs in non-smoking patients and is associated with EBV infection. Common treatments for tumors include multimodal therapy. The expression of PD-1 may be related to the prognosis of LELC, but more studies are needed to support further optimization of the treatment of LELC.

Keywords: Lymphoepithelioma-like carcinoma; treatment; prognosis; programmed death ligand-1 (PD-L1) expression

Submitted Aug 05, 2021. Accepted for publication Sep 24, 2021.

doi: $10.21037 /$ jtd-21-1369

View this article at: https://dx.doi.org/10.21037/jtd-21-1369

(C) Journal of Thoracic Disease. All rights reserved. 


\section{Introduction}

Lymphoepithelioma-like carcinoma (LELC) of the lung is a rare type of non-small cell lung cancer (NSCLC). In 2015, the World Health Organization (WHO) histological classification of lung neoplasm was reclassified as "other cancer and unclassified" and nasopharyngeal carcinoma (NPC) (1). It is a kind of epithelial tumor associated with Epstein-Barr virus (EBV), of which the histology and that of the former are indistinguishable $(2,3)$. The tumor is usually found in young, non-smoking Asian people. Patients with LELC NSCLC are diagnosed earlier and have a better prognosis than those with other types of NSCLC (4-6). It is characterized by nest-like or diffuse lamellar syncytic tumor cells with round or oval nuclei and prominent nucleoli with mixed infiltration of lymphocytes and plasma cells. The presence of EBV in tumor cells is critical for diagnosis. Epithelial growth factor receptor (EGFR) mutations and ALK rearrangements are uncommon in LELC; instead, and programmed death ligand-1 (PD-L1) expression is present in most cases $(7,8)$. The treatment of pulmonary lymphoepitheliomatoid carcinoma is a combination of surgery, chemotherapy, and radiotherapy, patients in the early and locally advanced stages are recommend to be treated with complete resection, for locally advanced disease, a combination of chemotherapy and radiotherapy is recommended.

Guangdong is the province with the highest incidence of EBV infection and NPC; however, oddly enough, LELC has not yet been reported. Over the past 10 years, our center and Zhaoqing First People's Hospital has treated several cases of LELC. Our study aimed to review the clinical features, diagnosis, staging, treatment, and prognosis of LELC and to determine if there are significant differences from published series. We also intended to assess the relationship with presence of EBV or PD-L1 expression and prognosis of LELC in our cohort.

We present the following article in accordance with the STROBE reporting checklist (available at https://dx.doi. org/10.21037/jtd-21-1369).

\section{Methods}

\section{Patients}

A total of 36 patients with LELC were diagnosed in the Fifth Affiliated Hospital of Sun Yat-sen University and Zhaoqing First People's Hospital from January 2014 to June 2021, which were confirmed by surgery or lung puncture biopsy. Patients with NPC or metastatic tumor of the viscera were excluded. The gender, age, tumor diameter, smoking status, EBV expression, PD-L1 expression, gene mutation status, and treatment history of the patients were collected. Other data such as re-biopsy after progression, genetic testing, and treatment plan were collected during the follow-up period. All procedures performed in this study involving human participants were in accordance with the Declaration of Helsinki (as revised in 2013). This study was approved by the Ethics Review Committee of the Fifth Affiliated Hospital of Sun Yat-sen University. All participants provided informed consent prior to participating in our study.

\section{Statistical methods}

In this study, SPSS 20.0 software (IBM Corp., Armonk, NY, USA) was used for descriptive statistical analysis of the perioperative clinicopathological characteristics of LELC. Survival and prognosis information collected during follow-up was analyzed using Kaplan-Meier method to calculate overall survival (OS) and progressionfree survival (PFS). Log-rank test was used to evaluate the significance of OS and PFS. Cox regression analysis was performed for univariate and multivariate factors affecting the prognosis. Statistical significance was considered when $\mathrm{P}<0.05$.

\section{Results}

\section{Clinical characteristics}

All 36 participants were Chinese, 20 of whom were female (56.5\%). The median age was 57 years (37-76 years). A total of 26 cases $(72.2 \%)$ were non-smokers. and 11 cases (30.5\%) had a family history of cancer. Some 9 participants (25\%) had stage I; 5 (13.8\%) had stage II; 5 (19.4\%) had stage IIIA; and 17 participants (47.2\%) had stage IIIB-IV (Table 1). There were 9 participants (25\%) with metastasis: mediastinal lymph node metastasis was the most common $(\mathrm{n}=7)$, followed by pleura $(\mathrm{n}=5)$, bone $(\mathrm{n}=2)$, and liver $(\mathrm{n}=1)$. Metastasis to organs other than those listed was not found in this study (Table 1).

\section{Molecular phenotype}

Because of the tumor's close association with EBV infection, the 2015 WHO classification requires the presence of EBV 
Table 1 Clinical characteristics of patients with pulmonary LELC

\begin{tabular}{|c|c|}
\hline Characteristics & Number (\%) \\
\hline \multicolumn{2}{|l|}{ Gender } \\
\hline Female & $20(55.5)$ \\
\hline Male & $16(45.5)$ \\
\hline \multicolumn{2}{|l|}{ Age, years } \\
\hline$<59$ & $15(41.7)$ \\
\hline$\geq 59$ & $21(58.3)$ \\
\hline \multicolumn{2}{|l|}{ Smoking history } \\
\hline Smokers & $10(27.8)$ \\
\hline Non-smokers & $26(72.2)$ \\
\hline \multicolumn{2}{|c|}{ Family history of cancers } \\
\hline Yes & $11(30.5)$ \\
\hline No & $25(69.4)$ \\
\hline \multicolumn{2}{|l|}{ T stage } \\
\hline 1 & $9(25)$ \\
\hline 2 & $10(27.8)$ \\
\hline 3 & $7(19.4)$ \\
\hline 4 & $10(27.8)$ \\
\hline \multicolumn{2}{|l|}{$\mathrm{N}$ stage } \\
\hline 0 & $12(33.3)$ \\
\hline 1 & $3(8.3)$ \\
\hline 2 & $8(22.2)$ \\
\hline 3 & $13(36.1)$ \\
\hline \multicolumn{2}{|l|}{ Stage } \\
\hline I & $9(25)$ \\
\hline II & $5(13.8)$ \\
\hline IIIA & $5(13.8)$ \\
\hline IIIB-IV & $17(47.2)$ \\
\hline \multicolumn{2}{|l|}{ Metastasis } \\
\hline Yes & $9(25)$ \\
\hline No & $27(75)$ \\
\hline \multicolumn{2}{|l|}{ p53 } \\
\hline Positive & $3(8.3)$ \\
\hline Negative & $22(61.1)$ \\
\hline Unknown & $11(30.6)$ \\
\hline
\end{tabular}

Table 1 (continued)
Table 1 (continued)

\begin{tabular}{|c|c|}
\hline Characteristics & Number (\%) \\
\hline \multicolumn{2}{|l|}{ EGFR mutations } \\
\hline Exon 19 & $1(2.7)$ \\
\hline Exon 21 & 0 \\
\hline No & $24(66.7)$ \\
\hline Unknown & $11(30.6)$ \\
\hline \multicolumn{2}{|l|}{ KRAS mutations } \\
\hline Yes & $0(0)$ \\
\hline No & $25(69.4)$ \\
\hline Unknown & $11(30.6)$ \\
\hline \multicolumn{2}{|c|}{ ALK rearrangements } \\
\hline Yes & $0(0)$ \\
\hline No & $25(69.4)$ \\
\hline Unknown & $11(30.6)$ \\
\hline \multicolumn{2}{|l|}{ PD-L1 } \\
\hline PD-L1 negative & $9(25.0)$ \\
\hline PD-L1 positive & 27 (75.0) \\
\hline \multicolumn{2}{|l|}{ EBV } \\
\hline EBV negative & $2(85.6)$ \\
\hline EBV positive & $34(94.4)$ \\
\hline
\end{tabular}

LELC, lymphoepithelioma-like carcinoma; T, tumor; N, node; $M$, metastasis; EGFR, epidermal growth factor receptor; PD-L1, programmed death-ligand 1; EBV, Epstein-Barr virus.

within the nuclei of the neoplastic cells in order to make a diagnosis of pulmonary LELC (9). In situ hybridization can be used to detect the presence of EBV for EBV-encoded small nuclear RNA (EBER). Tumor tissue of 34 participants (94.4\%) was positive for EBV (Table 1).

In terms of genetic testing, a study by Chang et al. showed that mutations in $\mathrm{p} 53$ and EGFR were not common in lung LELC (10). In this series, p53 mutations were found in only 3 of 36 cases $(8.3 \%)$ and EGFR mutations in 1 of 36 cases (2.7\%), which is L858R in exon 21 (Table 1).

The arrangement of ALK gene is another driver oncogene in lung cancer and is rarely found in lung LELC, and it was not found in this study. Immunohistochemistry revealed that $\mathrm{PD}-\mathrm{L} 1$ was expressed in the majority (27 cases, $75 \%$ ) of lung LELCs (Table 1). 

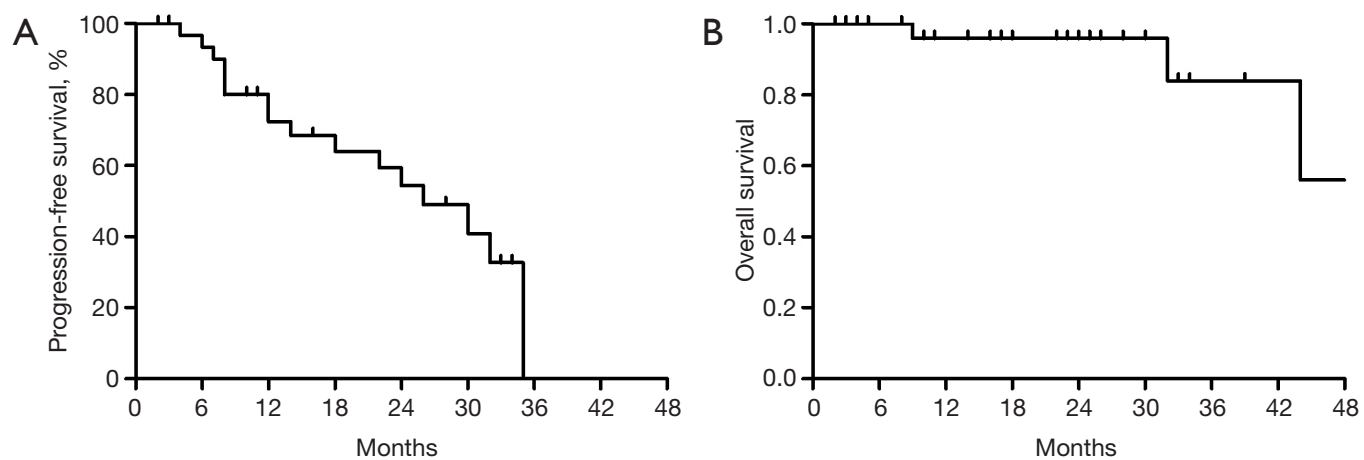

Figure 1 Survival analysis of pulmonary LELC patients. (A) PFS curve, median PFS was 26 months; (B) OS curve, median OS was 44 months. LELC, lymphoepithelioma-like carcinoma; PFS, progression-free survival; OS, overall survival.

\section{Treatment and outcomes of all participants}

As of 30 June 2021, complete survival data were available for all 36 participants. A total of 3 people were dead, and 33 others were still alive. During the study period, 16 cases of PFS occurred. The 1-year PFS rate was $68.6 \%$. The OS rates of 1 and 2 years was $96.0 \%$ and $84 \%$, respectively. The 3 deaths from tumor progression occurred at 32, 44, and 9 months, respectively. At the last evaluation on June 2021, the median follow-up time for PFS and OS was 26 and 44 months, respectively (Figure 1A,1B).

The majority of participants in our cohort received multimodal therapy. A total of 17 (47.2\%) participants received complete resection as initial treatment. A total of $5(13.8 \%)$ participants received adjuvant chemotherapy, including 2 in stage IIIB, 1 in stage IB, 1 in stage IIB, and 1 in stage IIIA. A solitary participant received gefitinib as adjuvant targeted therapy, whom was in stage pT1bN2M0, IIIA with EGFR L861R mutation.

In the course of palliative chemotherapy, 6 cases were given paclitaxel/nedaplatin, 3 were given paclitaxel/lobaplatin, 7 were given paclitaxel/carboplatin, 4 were given pemetrexed, and 2 participants were given docetaxel/lobaplatin. Paclitaxel/ nedaplatin + cisplatin/carboplatin (DDP/CBP) was the most commonly used scheme. One PD-L1 positive participant received sintilimab immunotherapy in combination with chemotherapy. Another participant was treated with an EGFR tyrosine kinase inhibitor (TKI) and was stable for 14 months after receiving gefitinib.

\section{Survival analysis of LELC}

As we know, a reading is considered positive if the level of $\mathrm{PD}-\mathrm{L} 1$ is greater than $1 \%$. Accordingly, we determined that the cut-off for differentiating PD-L1 of PFS was $1 \%$, so participants were classified as patients with negative expression of PD-L1 $(<1)$ or positive expression of PD-L1 $(\geq 1)$. Kaplan-Meier analysis showed that participants with positive PD-L1 expression tended to have longer PFS and OS times than those with negative PD-L1 expression. The 2-year OS for participants with negative expression of PD-L1 $(<1)$ or positive expression of PD-L1 $(\geq 1)$ was $78.9 \%$ and $100 \%$, respectively $(\mathrm{P}<0.001)$. The PFS for participants with negative expression of PD-L1 $(<1)$ or positive expression of PD-L1 $(\geq 1)$ was 26 months and 32 months, respectively $(\mathrm{P}=0.549)$ (Figure $2 A, 2 B)$. In univariate analysis, late node $(\mathrm{N})$ stage $(\mathrm{N} 2-3$ vs. $\mathrm{N} 0-1)$ and late metastasis (M) stage (M1 vs. M0) were not statistically different from PFS or OS (Figure $3 A, 3 B$ and Figure 4A,4B). Survival analysis was not performed for EGFR mutation, ALK rearrangement, and negative expression of EBV due to limited data.

\section{Conclusions}

Pulmonary LELC was first identified by Bégin et al. in 1987 in a 40-year-old non-smoking southeast Asian woman (11), and is currently classified as an additional and unclassified cancer under the 2015 WHO Classification Scheme (12). Primary lung LELC accounts for $0.9 \%(13,14)$ of all primary lung cancers and $0.4 \%$ (15) of all NSCLC. As reported previously, primary lung LELC mainly occurs in young and non-smoking Asians (16); however, in our study, $58.3 \%$ percent of the patients in this study were aged in their 60 s $(21 / 36 ; 58.3 \%)$. No significant difference between genders was observed.

The diagnosis of pulmonary LELC mainly depends on 

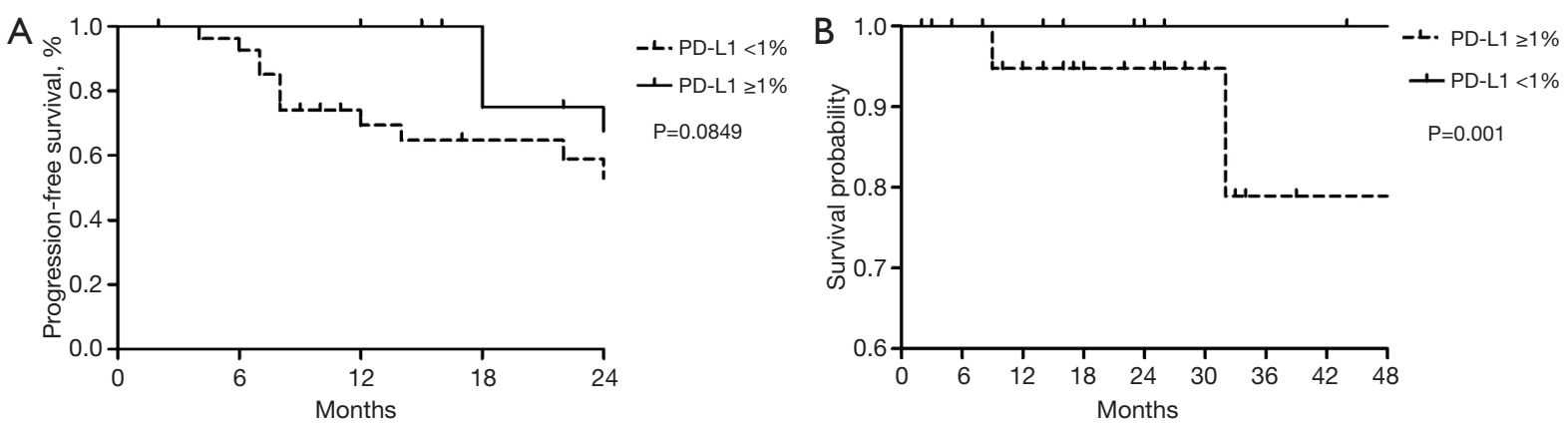

Figure 2 Survival analysis of pulmonary LELC patients with negative expression of PD-L1 (<1) or positive expression of PD-L1 ( $\geq 1$ ). (A) PFS curve, log-rank level of compared factors, $\mathrm{P}=0.0849$; (B) OS curve, log-rank level of compared factors, P=0.001. LELC, lymphoepithelioma-like carcinoma; PFS, progression-free survival; OS, overall survival; PD-L1, programmed death ligand 1.
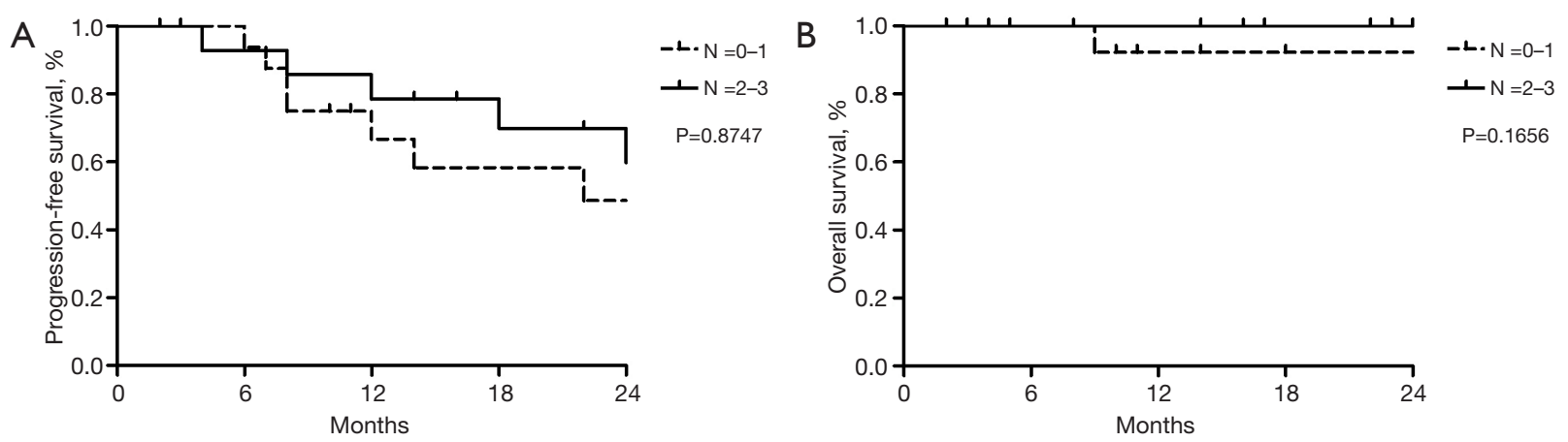

Figure 3 Survival analysis of pulmonary lymphoepithelioma-like carcinoma patients with late N stage (N2-3 vs. N0-1). (A) PFS curve, log-rank level of compared factors, $\mathrm{P}=0.8747$; (B) OS curve, log-rank level of compared factors, $\mathrm{P}=0.1656$. LELC, lymphoepithelioma-like carcinoma; PFS, progression-free survival; OS, overall survival; N, lymph node.
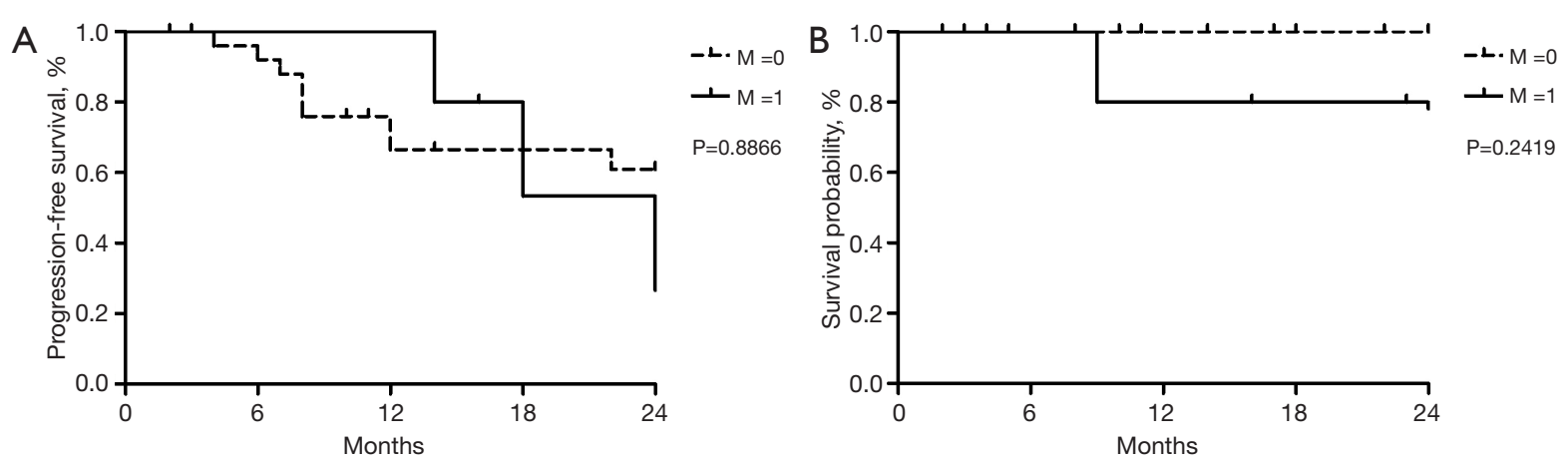

Figure 4 Survival analysis of pulmonary lymphoepithelioma-like carcinoma patients with late M stage (M1 vs. M0). (A) PFS curve, log-rank level of compared factors, $\mathrm{P}=0.8866$; (B) OS curve, log-rank level of compared factors, $\mathrm{P}=0.2419$. LELC, lymphoepithelioma-like carcinoma; PFS, progression-free survival; OS, overall survival; $M$, metastasis.

morphological features. Pulmonary LELC typically consists of undifferentiated cancer cells with unclear cytoplasmic boundaries arranged in syncytial plates and nests (14).
Primary lung LELC is associated with EBV infection. Since tumors are closely related to EBV infection, the WHO classification in 2015 required the presence of EBV 
in the nucleus of tumor cells to diagnose lung LELC (7). Previous research has shown that in Asia, pulmonary LELC is closely associated with $\mathrm{EBV}$ infection, which is present in about $67 \%$ of the patients from southern China, Taiwan, and Hong Kong (17). Our study retrospectively analyzed 36 patients with LELC from southeast China, and only 10 of those analyzed ( $<30 \%)$ had a history of smoking, indicating that primary lung LELC was the least associated with smoking compared to other lung cancers. In this study, 34 participants were positive in situ hybridization EBERs specimens, which highlights the correlation between EBV infection and pulmonary LELC.

Pulmonary LELC is a unique subtype of NSCLC with distinct genomic characteristics in addition to its unique histological appearance and persistent EBV infection (18). The clinical significance of gene mutations in lymphoepitheliomatoid carcinoma has not been determined and study of molecular characteristics is important for the potential of pulmonary LELC precision medicine. In terms of genetic testing, mutations in p53 and EGFR in lung LELC are not common. A study by Chang et al. showed that p53 mutations were found in only 3 of 46 cases (6.5\%) and EGFR mutations were found in 8 of 46 cases (17.4\%), but L858R mutations were not found in exon 21 (10). In the study of another group involving 42 cases, there was only 1 case of EGFR L858R mutation (2.4\%) (19). In our study, 25 participants had undergone gene testing and only 2 had EGFR mutations, suggesting that EGFR may not play an important role in the carcinogenesis of LELC. Rearrangement of the ALK gene is another driver oncogene in lung cancer and is rarely found in LELC $(20,21)$, only 1 case in Japan has reported EML4-ALK fusion gene in lung LELCs (22). Similarly, no patients with the ALK fusion gene mutation were identified in our study.

Programmed death receptor 1 (PD-1) is an important immunosuppressive molecule. It regulates the immune system and promotes self-tolerance by down regulating the response of the immune system to human cells and by inhibiting the inflammatory activity of $\mathrm{T}$ cells. PD-L1 is a ligand of PD-1. It is highly expressed in several cancers, including lung cancer. Its role in cancer immune escape has been well confirmed. In 2015, 2 case sequences showed lung LELCs (74.3\% to $75.8 \%$ ) expressing PDL1 immunohistochemically. Patients with high PD-L1 expression were reported to have a shorter disease-free survival compared to the PD-L1 negative group $(20,23)$. Interestingly, the opposite result was found in our study, wherein patients with positive PD-L1 expression tended to have longer PFS and OS time than those with negative PD-L1 expression.

Pulmonary LELC is treated in the same way as other NSCLC. Previous studies have shown that most patients with lung LELC are in the early and locally advanced stages of resectable lung disease, and complete resection is the main route to cure $(4,18)$. For locally advanced disease, a combination of chemotherapy and radiotherapy is recommended. The National Comprehensive Cancer Network (NCCN) guidelines (24) recommend the use of adjuvant chemotherapy in patients with stage IIa/B and Stage IIIA NSCLC whose tumors have been completely removed. Most case studies have shown that lung LELC is sensitive to chemotherapy. Previous studies have shown that surgery and adjuvant chemotherapy improve the prognosis of patients with early-stage LELC, while advanced LELC is more sensitive to chemotherapy and radiation (25). Platinum-based chemotherapy is considered as first-line treatment for advanced lung LELC. Adjuvant chemotherapy has been shown to significantly improve the outcome of stage IIIA LELC patients who undergo complete resection (6). In our study, most advanced patients received platinum-based chemotherapy. As LELC has similar biological characteristics to undifferentiated NPC, radiation doses of 5,000-7,000 cGy are often used (6).

Lung cancer is the leading cause of cancer-related death globally, and advanced lung cancer is generally considered to have a poor prognosis. The LELC is a rare subtype of NSCLC. Lung LELC patients tend to develop early, complete resection is possible, and prognosis is better than that of other NSCLC patients. Pulmonary LELC usually occurs in young and non-smoking patients and is associated with EBV infection. Common treatments for tumors include multimodal therapy with a combination of surgery, chemotherapy, and radiotherapy. The expression of PD-1 may be related to the prognosis of LELC, but more studies are needed to support further optimization of the treatment of LELC.

\section{Acknowledgments}

Funding: This work was funded by the Zhuhai Science and Technology Planning Project (ZH2202200004HJL) from the Fifth Affiliated Hospital of Sun Yat-sen University.

\section{Footnote}

Reporting Checklist: The authors have completed the 
STROBE reporting checklist. Available at https://dx.doi. org/10.21037/jtd-21-1369

Data Sharing Statement: Available at https://dx.doi. org/10.21037/jtd-21-1369

Conflicts of Interest: All authors have completed the ICMJE uniform disclosure form (available at https://dx.doi. org/10.21037/jtd-21-1369). The authors have no conflicts of interest to declare.

Ethical Statement: The authors are accountable for all aspects of the work in ensuring that questions related to the accuracy or integrity of any part of the work are appropriately investigated and resolved. All procedures performed in this study involving human participants were in accordance with the Declaration of Helsinki (as revised in 2013). This study was approved by the Ethics Review Committee of the Fifth Affiliated Hospital of Sun Yat-sen University. All participants provided informed consent prior to participating in the study.

Open Access Statement: This is an Open Access article distributed in accordance with the Creative Commons Attribution-NonCommercial-NoDerivs 4.0 International License (CC BY-NC-ND 4.0), which permits the noncommercial replication and distribution of the article with the strict proviso that no changes or edits are made and the original work is properly cited (including links to both the formal publication through the relevant DOI and the license). See: https://creativecommons.org/licenses/by-nc-nd/4.0/.

\section{References}

1. Travis WD, Brambilla E, Burke AP, et al. Introduction to The 2015 World Health Organization Classification of Tumors of the Lung, Pleura, Thymus, and Heart. J Thorac Oncol 2015;10:1240-2.

2. Pittaluga S, Wong MP, Chung LP, et al. Clonal EpsteinBarr virus in lymphoepithelioma-like carcinoma of the lung. Am J Surg Pathol 1993;17:678-82.

3. Iezzoni JC, Gaffey MJ, Weiss LM. The role of EpsteinBarr virus in lymphoepithelioma-like carcinomas. Am J Clin Pathol 1995;103:308-15.

4. Han AJ, Xiong M, Gu YY, et al. Lymphoepitheliomalike carcinoma of the lung with a better prognosis. A clinicopathologic study of 32 cases. Am J Clin Pathol 2001;115:841-50.
5. Ngan RK, Yip TT, Cheng WW, et al. Clinical role of circulating Epstein-Barr virus DNA as a tumor marker in lymphoepithelioma-like carcinoma of the lung. Ann N Y Acad Sci 2004;1022:263-70.

6. Liang Y, Wang L, Zhu Y, et al. Primary pulmonary lymphoepithelioma-like carcinoma: fifty-two patients with long-term follow-up. Cancer 2012;118:4748-58.

7. Sathirareuangchai S, Hirata K. Pulmonary Lymphoepithelioma-like Carcinoma. Arch Pathol Lab Med 2019;143:1027-30.

8. He J, Shen J, Pan H, et al. Pulmonary lymphoepitheliomalike carcinoma: a Surveillance, Epidemiology, and End Results database analysis. J Thorac Dis 2015;7:2330-8.

9. Lymphoepithelioma-like carcinoma. In: Travis WD, BrambillaE, Burke AP, et al. editors. WHO Classification of Tumours of the Lung, Pleura, Thymus and Heart. 4th ed. Lyon, France: International Agency for Research on Cancer; 2015:95-6.

10. Chang YL, Wu CT, Shih JY, et al. Unique p53 and epidermal growth factor receptor gene mutation status in 46 pulmonary lymphoepithelioma-like carcinomas. Cancer Sci 2011;102:282-7.

11. Bégin LR, Eskandari J, Joncas J, et al. Epstein-Barr virus related lymphoepithelioma-like carcinoma of lung. J Surg Oncol 1987;36:280-3.

12. Brambilla E, Pugatch B, Geisinger K, et al. WHO Classification of tumors-pathology \& genetics-tumours of the lung, pleura, thymus and heart. Lyon: IARC Press; 2004. Large cell carcinoma.

13. Chang YL, Wu CT, Shih JY, et al. New aspects in clinicopathologic and oncogene studies of 23 pulmonary lymphoepithelioma-like carcinomas. Am J Surg Pathol 2002;26:715-23.

14. Han AJ, Xiong M, Zong YS. Association of Epstein-Barr virus with lymphoepithelioma-like carcinoma of the lung in southern China. Am J Clin Pathol 2000;114:220-6.

15. Mo Y, Shen J, Zhang Y, et al. Primary lymphoepitheliomalike carcinoma of the lung: distinct computed tomography features and associated clinical outcomes. J Thorac Imaging 2014;29:246-51.

16. Castro CY, Ostrowski ML, Barrios R, et al. Relationship between Epstein-Barr virus and lymphoepithelioma-like carcinoma of the lung: a clinicopathologic study of 6 cases and review of the literature. Hum Pathol 2001;32:863-72.

17. Ho JC, Wong MP, Lam WK. Lymphoepithelioma-like carcinoma of the lung. Respirology 2006;11:539-45.

18. Chau SL, Tong JH, Chow C, et al.Distinct Molecular Landscape of Epstein-Barr Virus Associated Pulmonary 
Lymphoepithelioma-Like Carcinoma Revealed by Genomic Sequencing. Cancers (Basel) 2020;12:2065.

19. Wang L, Lin Y, Cai Q, et al. Detection of rearrangement of anaplastic lymphoma kinase (ALK) and mutation of epidermal growth factor receptor (EGFR) in primary pulmonary lymphoepithelioma-like carcinoma. J Thorac Dis 2015;7:1556-62.

20. Fang $W$, Hong $S$, Chen N, et al. PD-L1 is remarkably over-expressed in EBV-associated pulmonary lymphoepithelioma-like carcinoma and related to poor disease-free survival. Oncotarget 2015;6:33019-32.

21. Wong DW, Leung EL, So KK, et al. The EML4-ALK fusion gene is involved in various histologic types of lung cancers from nonsmokers with wild-type EGFR and KRAS. Cancer 2009;115:1723-33.

22. Ose N, Kawai T, Ishida D, et al. Pulmonary

Cite this article as: Sha Z, Wei Y, Gao T, Luo Y, Chen J, Li T, Hu L, Niu X, Lin Z, Lv W, Pei X. Clinical observation of pulmonary lymphoepithelioma-like carcinoma. J Thorac Dis 2021;13(10):5683-5690. doi: 10.21037/jtd-21-1369 lymphoepithelioma-like carcinoma with echinoderm microtubule-associated protein-like 4-anaplastic lymphoma kinase (EML4-ALK) fusion gene. Respirol Case Rep 2016;4:e00200.

23. Chang YL, Yang CY, Lin MW, et al. PD-L1 is highly expressed in lung lymphoepithelioma-like carcinoma: A potential rationale for immunotherapy. Lung Cancer 2015;88:254-9.

24. NCCN clinical practice guidelines in oncology NSCLC. V3.2011.

25. Huang CJ, Feng AC, Fang YF, et al. Multimodality treatment and long-term follow-up of the primary pulmonary lymphoepithelioma-like carcinoma. Clin Lung Cancer 2012;13:359-62.

(English Language Editor: J. Jones) 